
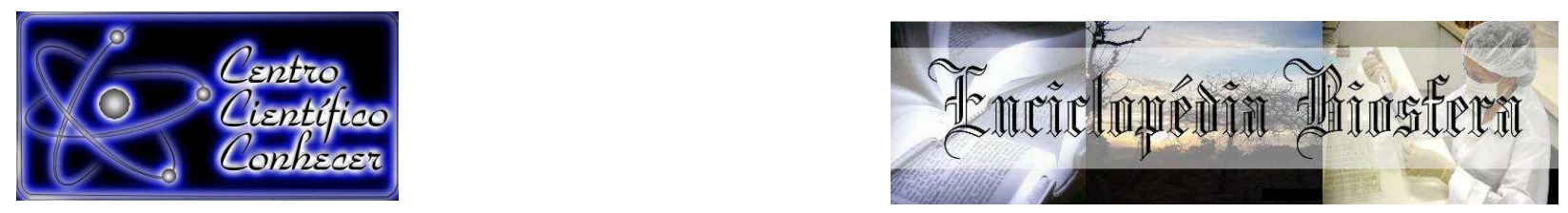

\title{
OBESIDADE E OSTEOARTRITE DE JOELHOS: PERFIL EPIDEMIOLÓGICO DE USUÁRIOS DO SISTEMA ÚNICO DE SAÚDE
}

Eduardo Paul Chacur ${ }^{1}$, Jean Ezequiel Limongi ${ }^{2}$, Fernando Leonardo Diniz ${ }^{3}$, Glória Lúcia Alves Figueiredo ${ }^{4}$, Cassiano Merussi Neiva ${ }^{5}$

${ }^{1}$ Doutor em Promoção da Saúde; Universidade de Franca - UNIFRAN, Professor ajunto e pesquisador no Instituto de Biotecnologia da Universidade Federal de Goiás

- UFG. eduardochacur@ufg.br / epcfisio@hotmail.com

${ }^{2}$ Doutor em Imunologia e Parasitologia Aplicadas pela Universidade Federal de

Uberlândia - UFU, Professor adjunto e pesquisador da Universidade Federal de Uberlândia - UFU.

${ }^{3}$ Doutor em Promoção da Saúde; Universidade de Franca - UNIFRAN, Professor e pesquisador da Faculdade de Patos de Minas - FPM.

Doutora em Enfermagem em Saúde Pública pela Universidade de São Paulo - USP,

Professora e pesquisadora da Universidade de Franca - UNIFRAN.

${ }^{2}$ Doutor em Biologia Funcional - UNICAMP; Professor adjunto e pesquisador,

Universidade Estadual Paulista Júlio de Mesquita Filho - UNESP; Professor e pesquisador do Programa de Pós-Graduação do Programa de Promoção de Saúde da Universidade de Franca - UNIFRAN; Professor e pesquisador da Faculdade de Medicina de Ribeirão Preto - UNAERP.

Recebido em: 08/04/2017 - Aprovado em: 10/06/2017 - Publicado em: 20/06/2017 DOI: 10.18677/EnciBio_2017A133

\section{RESUMO}

Dentre as doenças articulares, a Osteoartrite $(\mathrm{OA})$ é a mais comumente atendida na Atenção Básica. O objetivo deste estudo foi analisar a OA de joelhos em indivíduos adultos obesos atendidos em Unidades Básicas de Saúde e Unidades de Saúde da Família e os fatores associados. Participaram do estudo 226 indivíduos obesos, com idade entre 40 e 60 anos, sendo que 81 eram casos de Osteoartrite de joelho. A procura por consultas na especialidade ortopedia, depressão, idade cronológica e tempo de obesidade estiveram associadas à Osteoartrite. Concluí-se que atividades de promoção de saúde implementadas de forma contínua nas Unidades Básicas de Saúde e Unidades de Saúde da Família, bem como em outros espaços, pode reduzir sobremaneira o impacto desta doença na população atendida. Para aumentar a cobertura de atendimento, principalmente da população masculina, destaca-se a importância do horário de atendimento dos serviços públicos de saúde nestas unidades também no período noturno.

PALAVRAS-CHAVE: Atenção Primária à Saúde, Osteoartrite, Obesidade. 


\section{OBESITY AND KNEE OSTEOARTHRITIS: USERS EPIDEMIOLOGICAL PROFILE IN THE PUBLIC HEALTH SYSTEM \\ ABSTRACT}

Osteoarthritis $(O A)$ is the joint disorder most commonly treated in primary health care. The purpose of this study was to analyze knee OA and associated factors in obese adult patients treated at Basic Health Units (BHUs) and Family Health Units (FHUs). The study involved 226 obese 40 to 60-year-old patients, 81 of which suffered from knee OA. The demand for specialty consultations in orthopedics, depression, chronological age and duration of obesity was correlated with $\mathrm{OA}$. We concluded that health promotion activities implemented continuously at BHUs and FHUs, as well as at other facilities, can greatly reduce the impact this disease has on the population. We also emphasize the importance of these units offering nighttime public health services in order to increase the coverage of health services, especially of the male population.

KEYWORDS: Primary Health Care, Osteoarthritis, Obesity.

\section{INTRODUÇÃO}

A partir dos princípios do Sistema Único de Saúde (SUS), políticas são elaboradas de maneira a proporcionar o desenvolvimento de ações em saúde através de serviços em redes. As Unidades Básicas de Saúde (UBS) e Saúde da Família (SF) são os locais de acesso da população aos diagnósticos e às intervenções assistenciais básicas de saúde nos municípios brasileiros (SILVA \& SILVA, 2014).

A obesidade é um problema de saúde com proporção mundial, relacionada ao aumento de gordura no corpo devido ao excessivo consumo alimentar e baixo nível de atividade física. Várias são as causas, podendo enumerar os fatores socioeconômicos, culturais, psicológicos, ambientais e genéticos (REZENDE et al., 2013). Esta vem sendo tratada na literatura, como se o desenvolvimento fosse algo natural na sociedade e dependesse, principalmente, da vontade e comportamento do sujeito em relação à dieta e prática de atividade física. $\mathrm{Na}$ verdade os fatores etiológicos da obesidade estão enraizados na estrutura e funcionamento da sociedade capitalista e no sistema de organização social, sendo um problema social difícil de ser revertido (BARBIERI \& MELLO, 2012).

No Brasil, a prevalência de sobrepeso e obesidade aumentou no quadro populacional nacional progressivamente em pesquisas realizadas entre 1974-1975 e 2008-2009. Nos adultos, a prevalência de sobrepeso aumentou de $15,7 \%$ para $37,7 \%$ nos homens e de $20,7 \%$ a $31,1 \%$ em mulheres. Durante este mesmo período, a obesidade aumentou de $2,8 \%$ para $12,4 \%$ e de $8,0 \%$ para $16,9 \%$ em homens e mulheres, respectivamente (BERNARDO et al., 2012).

A OA é a doença articular mais comum. Envolve degeneração, processo inflamatório, dor, deformidades, alterações funcionais cotidianas como deslocamento e têm como um dos principais desencadeadores a obesidade (DE ROSIS et al., 2010). No Brasil a prevalência da Osteoartrite é de 16,9\%, responsável por 30 a $40 \%$ das consultas em ambulatórios de reumatologia, sendo ainda responsável por $7,5 \%$ de todos os afastamentos de trabalho e a quarta doença a determinar aposentadoria (6,2\%) (CARVALHO et al., 2008).

$A$ incidência da $O A$ aumenta de forma significativa entre a quarta e a quinta década de vida, na mulher no período da menopausa e a partir dos 50 anos nos homens, afetando $60 \%$ das pessoas com 65 anos ou mais e $80 \%$ 
daqueles com 75 anos ou mais (MICHAEL et al., 2010). Devido à alta prevalência da OA em indivíduos obesos, é necessário identificar fatores de risco incidentes para a etiologia e progressão da OA, compreender como a mesma se manifesta na população, e assim contribuir com a prevenção e o tratamento destes problemas visando a promoção da saúde.

Desta forma o objetivo desta pesquisa foi avaliar o perfil epidemiológico de indivíduos usuários do SUS para que estratégias de saúde possam ser melhor planejadas contribuindo com o bem estar da população e menores custos com o tratamento da $\mathrm{OA}$, diminuindo o número de afastamentos por este agravo, uma vez que os estudos que correlacionam a obesidade com a OA possuem resultados conflitantes (LEQUESNE et al., 1997; ISSA \& SHARMA, 2006; JANSSEN \& MARK, 2006; HAJIAN-TILAKI \& HEIDARI, 2007).

\section{MATERIAL E MÉTODOS}

Esta pesquisa se desenvolveu em um município de médio porte do Sudoeste Goiano com população estimada de 96.836 habitantes (IBGE 2014). A rede de Atenção Básica $(A B)$ do município é constituída por 12 Unidades Básicas de Saúde (UBS), e quatro Unidades de Saúde da Família (SF), distribuídas no município e em dois distritos.

Foram incluídos na pesquisa 226 voluntários, usuários das UBS e SF, com Índice de Massa Corpórea (IMC) acima de $30 \mathrm{Kg} / \mathrm{m}^{2}$, de ambos os sexos e idade entre 40 e 60 anos, divididos em dois grupos, o grupo 1, constituído por 81 indivíduos portadores de OA de joelho e o grupo 2, com 145 indivíduos sem $\mathrm{OA}$ de joelho. Os participantes foram recrutados por meio de convites fixados em forma de cartazes nas unidades de saúde, convites verbais dos pesquisadores na recepção das unidades de saúde e convites em domicílio por intermédio dos agentes comunitários de saúde (ACS) em suas respectivas áreas de abrangência. Foi adotado como critério de exclusão, indivíduos eutróficos e com sobrepeso, presença de lesões musculoesqueléticas na articulação do joelho que não fosse a $\mathrm{OA}$, ou que já sofreram fratura nos membros inferiores, presença de doenças imunológicas, déficit neurológicos e /ou cadeirantes.

O critério utilizado para o diagnóstico da obesidade foi o estabelecido pela Organização Mundial da Saúde para a classificação do IMC, produto da divisão do peso corporal pela altura ao quadrado $\left(P / A^{2}\right)$ (WHO, 1995). Foi calculada a relação cintura-quadril, sendo classificado o tipo de obesidade, pela divisão do perímetro abdominal entre a última costela e a crista ilíaca pelo perímetro dos quadris no nível dos trocânteres femorais com o indivíduo em posição ortostática, por meio da mensuração pela fita métrica. A distribuição androide (central) definida pelos índices superiores a 0,9 em mulheres e 1 nos homens. Outra medida utilizada na avaliação foi a circunferência abdominal (CA), determinada pela mensuração da maior circunferência do abdome, sendo considerada aumentada quando o valor foi $\geq$ a $88 \mathrm{~cm}$ para mulheres $\mathrm{e} \geq$ a 102 para homens (JANSSEN \& MARK, 2006).

O questionário de Baecke (FLORINDO et al., 2004) foi aplicado para avaliar - nível de atividade física habitual dos voluntários. Este fornece escores caracterizados por atividades ocupacionais, exercícios físicos e atividades de lazer e locomoção, realizadas no último ano. O questionário não fornece um escore final em categorias. Para se obter este, foram utilizados os critérios usados por TURI et al., 2014) em que a pontuação total do questionário foi 
subdividida em quartis como segue: quartil inferior $\left(1^{\circ}\right)$, sedentários; quartis intermediários ( $2^{\circ}$ e $3^{\circ}$ ), moderadamente ativos; e quartil superior (4ํำ, ativos.

Primeiramente os participantes foram selecionados apenas por critérios de idade e IMC. Em seguida foram avaliados pelos pesquisadores, sendo realizado exame físico como inspeção e palpação da articulação do joelho. De acordo com os antecedentes históricos e o exame clínico, os indivíduos foram separados em grupo OA (possíveis portadores de OA de joelhos) e grupo S/OA (possíveis não portadores de $\mathrm{OA}$ ). $\mathrm{O}$ diagnóstico da $\mathrm{OA}$ foi realizado clinicamente e por meio de radiografia ortostática (com exceção dos que já possuíam o exame por um período menor que um ano) em incidências anteroposterior e perfil da articulação do joelho dos voluntários, realizado por um ortopedista.

A coleta de dados ocorreu nas próprias Unidades Básicas de Saúde e também nas residências dos participantes em visitas indicadas pelos ACS das unidades. A pesquisa foi aprovada pelo Comitê de Ética em Pesquisa em Humanos da Universidade de Franca, sob o protocolo $n^{0} 565863$. Os voluntários foram orientados sobre os objetivos e procedimentos da pesquisa e assinaram o Termo de Consentimento Livre e Esclarecido. Não houve conflitos de interesses.

\section{ANÁLISE ESTATÍSTICA}

A análise dos dados foi executada utilizando o programa EPI INFO versão 3.5.4 (www.cdc.gov/epiinfo). Nas comparações para duas proporções foi utilizado o Teste Exato de Fisher ou teste Qui-quadrado. Em comparações de variáveis com mais de duas categorias foi utilizado a Regressão Logística. Para as comparações das variáveis contínuas, foi utilizado o teste T de Student ou o teste não paramétrico Wilcoxon-Mann-Whitney. Para a verificação da normalidade dos dados foi utilizado o Teste de Lilliefors. Como medida de associação foi utilizada a Odds Ratio (OR) com intervalo de confiança de 95\%. O nível de significância adotado foi de $5 \%$.

\section{RESULTADOS}

Foram avaliados participantes das 12 UBS e quatro SF do município estudado. A UBS que contribuiu com maior parte da amostra do Grupo 1 foi $12,3 \%$ e a menor $4,9 \%$, já no grupo 2 a que contribui com maior número foi $13,8 \%$ e a menor $4,8 \%$. A consulta médica foi o serviço clínico de saúde mais citado pelos pacientes, perfazendo um total de $92,6 \%$ no Grupo 1 e 91,7\% no Grupo 2. Os demais serviços clínicos de saúde como a Fisioterapia, Nutrição e Psicologia, apresentaram baixa utilização pelos usuários, que pode sugerir uma menor disponibilidade destes serviços de saúde para a população e consequente não utilização de serviços multiprofissionais que poderiam proporcionar maior integralidade no cuidado à saúde da população.

Ao analisar as especialidades médicas mais utilizadas pelos participantes do estudo, a clínica geral foi a mais relatada com $42 \%$ no Grupo 1 e 55,2\% no Grupo 2. A ortopedia representou 18,5\% no Grupo 1 e 7,6\% no 2. Observou-se que, indivíduos obesos com $\mathrm{OA}$ procuram 2,7 vezes mais a especialidade da Ortopedia do que as demais especialidades médicas em relação a indivíduos obesos sem OA. 
TABELA 1. Análise bivariada entre variáveis categóricas de participantes do grupo $1 \mathrm{e}$ grupo 2

\begin{tabular}{|c|c|c|c|c|c|c|c|}
\hline & \multicolumn{2}{|c|}{ Grupo 1} & \multicolumn{2}{|c|}{ Grupo 2} & \multirow{2}{*}{$\begin{array}{l}\text { Valor } \\
\text { de p }\end{array}$} & \multirow{2}{*}{ Teste } & \multirow{2}{*}{ OR (IC 95\%) } \\
\hline & $\mathbf{N}$ & & & & & & \\
\hline \multicolumn{8}{|l|}{$\begin{array}{l}\text { Especialidade } \\
\text { médica }\end{array}$} \\
\hline Ortopedia & 15 & 57,7 & 11 & 42,3 & $0,02^{*}$ & Qui-quadrado & $2,75(1,19-6,51)$ \\
\hline Outras & 66 & 33,0 & 134 & 67,0 & & & \\
\hline \multicolumn{8}{|l|}{ Depressão } \\
\hline Sim & 20 & 51,3 & 19 & 48,7 & $0,04^{*}$ & Qui-quadrado & $2,16(1,07-4,40)$ \\
\hline Não & 61 & 32,6 & 126 & 67,4 & & & \\
\hline \multicolumn{8}{|l|}{$\begin{array}{l}\text { Nivel de Atividade } \\
\text { Fisica }\end{array}$} \\
\hline Ativo & 18 & 45,0 & 22 & 55,0 & & $\begin{array}{l}\text { Regressão } \\
\text { logística }\end{array}$ & 1 \\
\hline Moderadamente ativo & 42 & 32,3 & 88 & 67,7 & 0,14 & & $0,58(0,28-1,20)$ \\
\hline Sedentário & 21 & 37,5 & 35 & 62,5 & 0,46 & & $0,73(0,32-1,67)$ \\
\hline
\end{tabular}

Os voluntários do grupo 1 eram significantemente mais velhos e mais tempo obesos quando comparados com o grupo 2. Todos os participantes apresentaram circunferência abdominal maior que $97 \mathrm{~cm}$ e $R C Q>0,81$. As variáveis antropométricas dos participantes dos dois grupos estão apresentadas na tabela 2.

TABELA 2. Análise bivariada das variáveis numéricas entre os participantes do grupo 1 e grupo 2.

\begin{tabular}{|c|c|c|c|c|c|}
\hline & \multicolumn{2}{|c|}{$\begin{array}{c}\text { Grupo 1 } \\
(\mathrm{N}=81)\end{array}$} & \multicolumn{2}{|c|}{$\begin{array}{l}\text { Grupo } 2 \\
(\mathrm{~N}=145)\end{array}$} & \multirow[t]{2}{*}{ Valor de $\mathrm{p}^{*}$} \\
\hline & Média & DP & Média & DP & \\
\hline Idade (anos) & 51,6 & $\pm 5,5$ & 49,4 & $\pm 6,1$ & 0,01 \\
\hline Altura (m) & 1,58 & $\pm 0,07$ & 1,58 & $\pm 0,08$ & 0,77 \\
\hline Peso (Kg) & 89,8 & $\pm 15,2$ & 87,2 & $\pm 15,6$ & 0,23 \\
\hline IMC $\left(\mathrm{Kg} / \mathrm{m}^{2}\right)$ & 35,74 & \pm 4.9 & 34,72 & $\pm 5,1$ & 0,14 \\
\hline $\mathrm{RCQ}$ & 0,88 & $\pm 0,07$ & 0,88 & $\pm 0,06$ & 0,79 \\
\hline $\mathrm{CA}(\mathrm{cm})$ & 111,35 & $\pm 13,2$ & 109,10 & $\pm 11,88$ & 0,19 \\
\hline Tempo de obesidade (anos) & 13,3 & $\pm 7,1$ & 104 & $\pm 7,2$ & $<0,001$ \\
\hline
\end{tabular}

IMC: Índice de massa corporal; CA: Circunferência Abdominal; RCQ:Relação Cintura Quadril;

A ocorrência de depressão associada foi mais frequente entre os indivíduos do grupo $1(24,7 \%)$ quando comparados com o grupo $2(13,1 \%)$ (tabelas 1 e 3$)$. Esta foi investigada por meio de questionamento auto-referindo sobre comorbidades associadas à obesidade. No entanto, apenas $4,9 \%$ e $4,8 \%$ dos pacientes dos grupos 1 e 2 respectivamente têm acompanhamento psicológico. 
TABELA 3 - Frequência das variáveis físicas e comportamentais dos participantes.

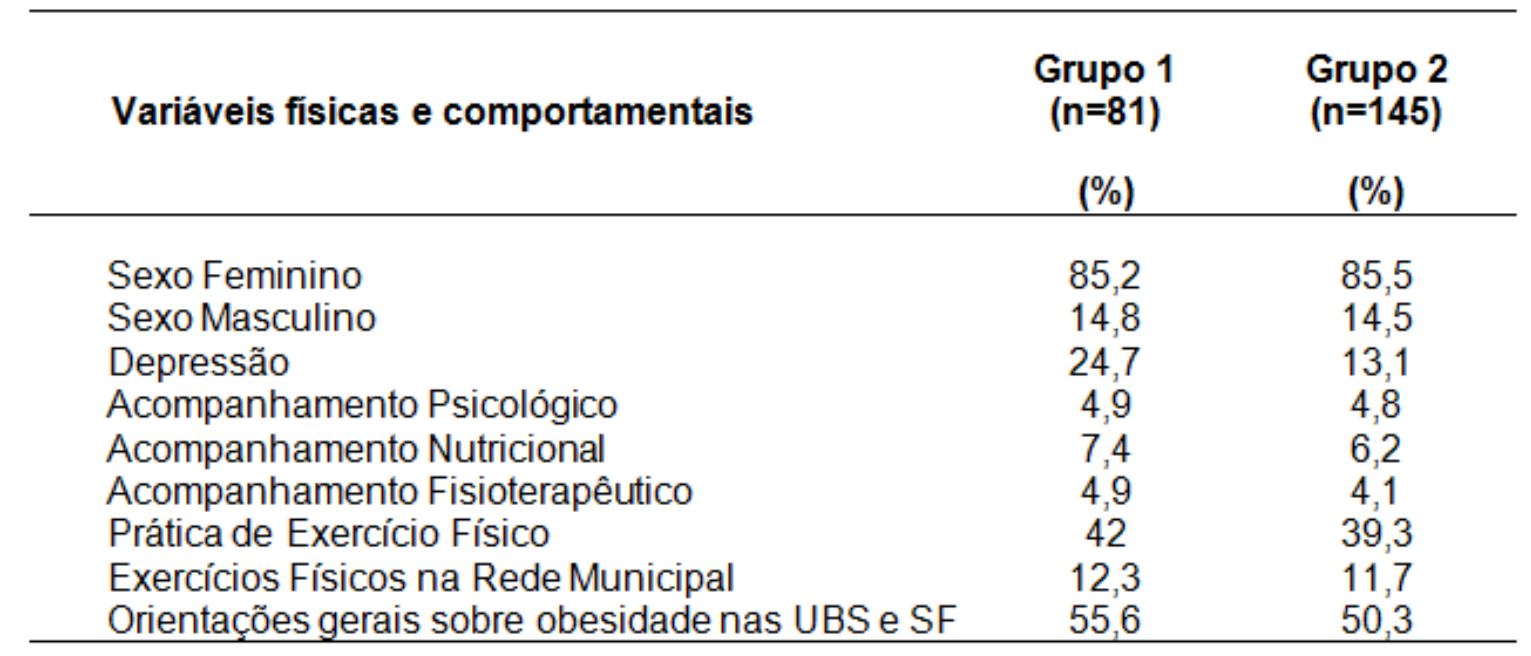

Quanto ao nível de atividade física, ambos os grupos apresentaram maior número de indivíduos moderadamente ativos, porém, o grupo 1 apresentou um maior número de indivíduos ativos em relação ao Grupo 2 (tabela 4). Porém, não houve diferença significativa na comparação das categorias de atividade física entre os grupos (tabela 4).

TABELA 4 - Nível de Atividade Física dos voluntários do estudo segundo interpretação do questionário de Baecke et al., (FLORINDO et al., 2004) e com escores segundo URI et al., (2014).

\begin{tabular}{lcc}
\hline Nivel de Atividade Física & Grupo 1 (n=81) & Grupo 2 (n=145) \\
\hline Sedentários & $25,9 \%$ & $24,1 \%$ \\
Moderadamente ativos & $51,9 \%$ & $60,7 \%$ \\
Ativos & $22,2 \%$ & $15,2 \%$ \\
\hline
\end{tabular}

A prática de exercícios físicos foi maior entre os indivíduos do grupo $1 \mathrm{com}$ $42 \%$ da amostra, sendo que destes, somente $12,3 \%$ realizam na rede municipal. Já no grupo 2 39,3\% praticam exercício físico e 11,7\% destes realizam na rede municipal. (tabela 3) A caminhada foi a modalidade esportiva mais citada pelos participantes dos dois grupos, representando $76,5 \%$ no grupo 1, destes, $11,8 \%$ associam com a prática de hidroginástica. No grupo 2 observou-se que 72,1\% praticam caminhada, e 3,5\% associaram com a prática de hidroginástica.

\section{DISCUSSÃO}

As mulheres representaram $84,9 \%$ da amostra total, o que demonstra uma maior procura e utilização dos serviços de saúde de atenção básica e consequentemente leva ao maior registro de incidência da OA no gênero feminino. Esses resultados estão de acordo com os apresentados por MALTA et al., (2009) que observaram maior incidência de doenças crônicas não transmissíveis no sexo feminino. A procura pelos serviços de saúde proporciona maior oportunidade 
de diagnóstico de agravos à saúde, já os homens em idade produtiva, que estão em maior número ainda alocados no mercado de trabalho, encontram dificuldades na busca por atendimento na atenção básica, devido ao horário de funcionamento das UBS, tendendo assim a optar por serviços de pronto atendimento hospitalar ou consultórios particulares em caráter emergencial (GOMES et al., 2007; BRASIL, 2007; OLIVEIRA et al., 2015).

Essa situação faz com que a atenção básica à saúde adquira uma concepção eminentemente feminina, sendo imperativo o desenvolvimento de estratégias para maior adesão do gênero masculino em ações de prevenção primária e secundária (DOMINGUES et al., 2012). Dentre os serviços de saúde disponíveis nas unidades de saúde, as consultas médicas foram os mais utilizados $(90 \%)$, o que pode estar relacionado à maior disponibilidade desta nas UBS por ser especialidade básica na atenção primária. Foi relatada baixa utilização dos demais serviços clínicos como a fisioterapia e a nutrição, que estão diretamente relacionadas ao tratamento da obesidade e da OA.

Nesta pesquisa notou-se que somente $4,9 \%$ dos obesos portadores de OA realizam fisioterapia e apenas 7,4\% fazem acompanhamento nutricional, o que demonstra que 0 tratamento dos pacientes não acontece de forma multiprofissional (REZENDE \& CAMPOS, 2013). MUSUMECl et al., (2015) concluíram em estudo que dietas alimentares destinadas a redução de peso, atividade física e a fisioterapia são essenciais no tratamento da OA e devem ser estimuladas aos portadores da doença.

O serviço clínico de ortopedia é a especialidade médica mais relacionada ao diagnóstico e ao tratamento da $\mathrm{OA}$ dentre as especialidades citadas pelos participantes. Os participantes do Grupo 1 relataram maior utilização da especialidade em relação aos do Grupo 2. A SF é a estratégia mais importante da atenção primária, responsável por prevenção de enfermidades e riscos mais prevalentes na comunidade além do atendimento básico de enfermos. A ortopedia não se enquadra como especialidade da atenção básica por ser de cunho principalmente curativo. Sendo assim, as SF e UBS, apoiadas pelos Núcleos de Apoio à Saúde da Família seriam essenciais para ações de promoção da saúde e prevenção da $O A$ de forma integral, deixando a especialidade da ortopedia de ser porta de entrada para o tratamento da OA (LIMONGE et al., 2008).

Maiores índices de IMC foram verificados entre os pacientes do grupo 1. Para ANJOS (2013) o IMC apresenta boa correlação com a gordura corporal. Na amostra total desta pesquisa foram identificados $13 \%$ dos participantes com IMC > que $40 \mathrm{Kg} / \mathrm{m}^{2}$. SOWERS \& CARRIE (2010) demonstram associação entre o IMC e $O A$ de joelhos devido principalmente ao fato que a $O A$ dos joelhos tem grande relação com a situação metabólica altamente inflamatória encontrada na obesidade.

A CA não mostrou associação com a presença de $O A$, porém foram registrados valores acima do aceitável em todos os pacientes, o que corrobora com os estudos de HOLANDA et al., (2011) que também observaram CA aumentada em indivíduos com idade entre 50 e 59 anos. MELLER et al., (2006) concluíram em estudo que a elevada prevalência de mulheres com IMC elevado, implica na necessidade de políticas públicas de saúde que visem não somente a prevenção e o tratamento do excesso de peso, mas também da obesidade abdominal, a fim de promover o estado nutricional adequado e reduzir as prevalências das doenças associadas. Neste estudo, todos os voluntários apresentaram CA maior que $97 \mathrm{~cm}$, que associado aos elevados valores de IMC observados, confere alto risco a problema de saúde associados a essa parcela da população. Segundo VELOSO 
\& SILVA (2010) o acúmulo de adiposidade na região abdominal é fator de risco para doenças endócrinas, metabólicas e cardiovasculares, mesmo quando o IMC está dentro dos limites da normalidade.

Outra variável antropométrica importante que demonstrou valores aumentados foi a RCQ. Todos os voluntários da pesquisa apresentaram esta razão maior que 0,81 , e $37 \%$ da amostra total apresentaram esta razão maior que 0,9 , o que representa de acordo com a OMS (1995) um risco muito alto de complicações cardiovasculares para a população feminina e risco alto para a masculina. Esta, não apresentou relação com 0 desenvolvimento da OA nesta pesquisa, mas é considerada uma medida de rotina para a educação em saúde e diminuição do impacto da OA de joelho na saúde pública (OGUMBODE, 2014).

A idade foi uma variável que mostrou associação estatisticamente significante em relação à presença de $\mathrm{OA}(\mathrm{p}=0,01)$, com maiores idades entre os pacientes do grupo 1 , esse dado corrobora com os resultados de pesquisas na mesma temática (CHACUR et al., 2010; LEITE et al., 2011). O aumento da idade aumenta à incidência de $\mathrm{OA}$ em todas as articulações. Quando se trata da articulação do joelho, é importante a relação com a capacidade funcional. A dor faz com que 0 indivíduo evite atividades que a intensifique, sendo o joelho uma articulação de transição dos membros inferiores que compromete a prática de exercícios envolvidos com 0 deslocamento e consequente diminuição do condicionamento físico e da capacidade funcional (DE ROSIS et al., 2010).

O tempo de obesidade em anos também se associou com a presença de OA de joelhos $(p<0,001)$ o que também reafirma resultado de um estudo na área (CHACUR et al., 2010). A associação de depressão e AO foi significativa neste estudo $(p=0,04)$. HARRIS et al., (2015) em estudo relataram que a OA é uma condição clínica e insidiosa que impacta significativamente no funcionamento físico e mental do indivíduo.

Ao se comparar o diagnóstico de depressão entre os grupos estudados, observou-se que indivíduos obesos portadores de depressão têm 2,16 vezes mais chance de ter OA associada que indivíduos obesos sem depressão. Apesar de a depressão ser uma comorbidade importante na população estudada foi verificado que apenas $9 \%$ da amostra total fazem acompanhamento psicológico o que reafirma que o tratamento da OA não ocorre de forma multiprofissional.

CHEN et al., (2013) em seus achados demonstraram que pessoas com OA de joelho e história de queda tem maior risco de sintomas depressivos que indivíduos que nunca sofreram quedas e que não possuem $O A$ de joelhos. Mudanças no estilo de vida como: inclusão de exercícios e redução de comportamento sedentário contribui para minimizar eficazmente fatores de risco para doenças crônicas. Dentre as melhorias na saúde geral de idosos, inclui-se redução dos sintomas depressivos, das medidas antropométricas, além de aumento na aptidão física (BRANCO et al., 2015).

A caminhada foi a modalidade esportiva mais praticada entre os participantes deste estudo perfazendo um total de $72,6 \%$ da amostra total. Segundo DEL DUCA et al., (2014) em estudo sobre a prática de atividades físicas no lazer a preferência pela caminhada se dá pela possibilidade de prática conjunta entre indivíduos que vivem acompanhados. Ao término da coleta, os participantes que praticavam caminhadas receberam orientações gerais dos pesquisadores sobre como realizar uma caminhada de forma eficiente.

A atividade física e obesidade são dois indicadores importantes para a saúde individual e da população (PEREIRA \& SILVA, 2011). Neste estudo foi observado 
que $25 \%$ dos participantes são sedentários. A amostra se encontra abaixo da média nacional divulgada pela OMS em 2011, em que 48,6\% dos indivíduos adultos foram considerados inativos fisicamente.

\section{CONCLUSÃO}

A OA é um problema de saúde pública que deve ser tratada de forma multiprofissional. $O$ atendimento voltado principalmente às consultas médicas, sem acompanhamento de outros profissionais impede que a OA, assim como outras doenças no âmbito do SUS, sejam tratadas de forma integral e resolutiva.

Atividades de promoção de saúde implementadas de forma contínua e bem orientada na atenção básica pode contribuir sobremaneira não somente no tratamento da $O A$ e obesidade, mas também em outras comorbidades associadas. Além disso, a busca ativa em residências como preconiza a Saúde da Família e ampliação do horário de atendimento dos serviços públicos de saúde das UBS e SF além do horário comercial seriam medidas de fundamental importância para aumentar a assistência à saúde da população.

\section{AGRADECIMENTOS}

À Secretaria Municipal de Saúde do município de Catalão, em especial às Coordenadoras das UBS e SF e aos Ortopedistas Dr. Joelberth Moraes Cindra e Dr. Flávio Malagoni Buiatti pelo apoio à realização dos exames de Raios-X e classificação dos mesmos.

\section{REFERÊNCIAS}

ANJOS, L.A. Diagnóstico de obesidade e determinação de requerimentos nutricionais: desafios para a área de Nutrição. Ciência e Saúde Coletiva 18(2): 29494, 2013. Disponível em: <http://www.scielo.br/pdf/csc/v18n2/01.pdf>. Acessado em 31 de maio de 2017.

BARBIERI, A. F.; MELLO, R. A. As causas da obesidade: uma análise sob a perspectiva materialista histórica. Revista da Faculdade de Educação Física da UNICAMP10(1):133-53,2012. Disponível em: https://periodicos.sbu.unicamp.br/ojs/index.php/conexoes/article/view/8637693/5384>. Acessado em 31 de maio de 2017.

BERNARDO, C.O.; BOING, A. F.; VASCONCELOS, F. A. G.; PERES, K. G.; PERES, M. A. Association between tooth loss and obesity in Brazilian adults: a populationbased study. Revista Saúde Pública 46(5): 834-42, 2012. Disponível em: < http://dx.doi.org/10.1590/S0034-89102012000500010>.

BRANCO, J.C.; JANSEN, K.; SOBRINHO, J. T.; CARRAPATOSO, S.; SPESSATO, B.; CARVALHO, J.; MOTA, J.;SILVA, R. A. Physical benefits and reduction of depressive symptoms among the elderly: Results from the Portuguese "National Walking Program". Ciência e saúde coletiva. 20(3): 789-95, 2015. doi.org/10.1590/141381232015203.09882014

BRASIL, Ministério da Saúde. Secretaria de Vigilância em Saúde. Departamento de 
Análise de Situação em Saúde. Perfil da Saúde no homem. In: Brasil. Ministério da Saúde (MS). Saúde Brasil 2007: uma análise da situação de Saúde. Brasília: MS 509-36, 2007. Disponível em: < http://bvsms.saude.gov.br/bvs/publicacoes/saude_brasil_2007.pdf>. Acessado em 31 de maio de 2017.

CARVALHO, M.A.P.; LANNA, C.C.D.; BÉRTOLO, M.B. Reumatologia diagnóstico e tratamento. Rio de Janeiro: Guanabara Koogan, 245-53, 2008.

CHACUR, E.P.; SILVA, L. O.; LUZ, G. C. P.; KAMINICE, F. D.; CHEIK, N. C. Avaliação antropométrica e do ângulo quadricipital na osteoartrite de joelho em mulheres obesas. Fisioterapia e Pesquisa. 17(3): 220-24, 2010. Disponível em: < http://www.scielo.br/pdf/fp/v17n3/06.pdf>. Acessado em 31 de maio de 2017.

CHEN, Y; MO, F; YI, K; MORRISON, W; MAO, Y. Association between mental health and fall injury in Canadian immigrants and non-immigrants. Accident analysis and prevention. v. 59, n. 1, p. 221-26, 2013. Disponível em: <http://dx.doi.org/10.1016/j.aap.2013.06.005>.

DE ROSIS, R.G.; PAULO, S.M.; KAIRALLA, M. Osteoartrite: avaliação clínica e epidemiológica de pacientes idosos em instituição de longa permanência, Revista Brasileira de Clínica Médica. 8(2): 101-8, 2010. Disponível em: < http://files.bvs.br/upload/S/1679-1010/2010/v8n2/a003.pdf>. Acessado em 31 de maio de 2017.

DEL DUCA, G.F.; NAHAS, M. V.; HALLAL, P. C.; PERES, K. G. Atividades físicas no lazer entre adultos de Florianópolis, Santa Catarina, Brasil: estudo populacional sobre as características das práticas e de seus praticantes. Ciência Saúde Coletiva [online]. 19(11), 4595-604, 2014. Disponível em: <DOI: 10.1590/1413812320141911.16732013>.

DOMINGUES, P.S.; DAHER, D.V.; PINTO, A.A. Health education as a possibility for health promotion of men. Journal of Nursing UFPE. 6(12):3034, 2012. Disponível em: < DOI: 10.5205/reuol.2265-25464-1-LE.0612201221>.

FLORINDO, A. A.; LATORRE, M. R. D. O.; JAIME, P. C.; TANAKA, T.; ZERBINI, C. A. F. Metodologia para a avaliação da atividade física habitual em homens com 50 anos ou mais. Revista Saúde Pública. 38(2): 307-314, 2004. Disponível em: < http://www.revistas.usp.br/rsp/article/viewFile/31716/33612>. Acessado em 31 de maio de 2017.

GOMES, R.; NASCIMENTO, E. F.; ARAUJO, F. C. Por que os homens buscam menos os serviços de saúde do que as mulheres? As explicações de homens com baixa escolaridade e homens com ensino superior. Cadernos de Saúde Pública. 23(3): 565- 74, 2007. Disponível em: < http://www.scielo.br/scielo.php?pid=S0102311 X2007000300015\&script=sci_abstract\&tlng=pt>. Acessado em 31 de maio de 2017.

HAJIAN-TILAKI, K. O.; HEIDARI, B. Prevalence of obesity, central obesity and the associated factors in urban population aged 20-70 years, in the north of Iran: a 
population-based study and regression approach. Obesity Reviews. 8(1): 3-10, 2007. Disponível em: < DOI: 10.1111/j.1467-789X.2006.00235.x

HARRIS, M. L.; BYLES, J. E.; SIBBRITT, D.; LOXTON D. "Just Get on with It": Qualitative Insights of Coming to Terms with a Deteriorating Body for Older Women with Osteoarthritis. PLOS ONE. 10(3), 1-15, 2015. Disponível em: < DOI:10.1371/journal.pone.0120507>.

HOLANDA, L. G. M.; MARTINS, M. C. C.; FILHO, M. D. S.; CARVALHO, C. M. R. G.; ASSIS, R. C.; LEAL, L. M. M., et al., Excesso de peso e adiposidade central em adultos de Teresina-PI. Revista da Associação Médica Brasileira. 57(1): 50-55, 2011. Disponível em: < http://www.scielo.br/pdf/ramb/v57n1/v57n1a16>. Acessado em 31 de maio de 2017.

ISSA, S. N.; SHARMA, L. Epidemiology da osteodistrofia: um update. Current Rheumatology. 6(1): 7-15, 2006. Disponível em: < https://www.ncbi.nlm.nih.gov/pubmed/16515759>. Acessado em 31 de maio de 2017.

JANSSEN, I.; MARK, A. E. Separate and combined influence of body mass index and waist circumference on arthritis and Knee osteoarthritis. International Journal of Obesity. 1(6): 1-6, 2006. Disponível em: < http://library.nuft.edu.ua/ebook/file/0803287a.pdf>. Acessado em 31 de maio de 2017.

LEITE, A. A.; COSTA, A. J. G.; LIMA, B. A. M.; PADILHA, A. V. L.; ALBUQUERQUE, E. C.; MARQUES, C. D. L. Comorbidades em pacientes com osteoartrite: frequência e impacto na dor e na função física. Revista Brasileira de Reumatologia. 51(2), 118-23, 2011. Disponível em: < http://www.scielo.br/pdf/rbr/v51n2/v51n2a02>. Acessado em 31 de maio de 2017.

LEQUESNE, M. G.; DANG, N.; LANE, N. Sport practice and osteoarthritis of the limbs. Osteoarthritis and Cartilage. 5(2): 75-6, 1997. Disponível em: < http://www.oarsijournal.com/article/S1063-4584(97)80001-5/pdf>. Acessado em 31 de maio de 2017.

LIMONGI, J. E.; MENEZES, E. C.; MENEZES, A. C. Vigilância em Saúde no Programa Saúde da Família. Hygeia. Revista Brasileira de Geografia Médica e da Saúde. 1(4), p. 35-44, 2008.

MALTA, D. C.; CASTRO, A. M. GOSCH, C. S.; CRUZ, D. K. A.; BRESSAN, A.; NOGUEIRA, J. D.; MORAIS-NETO, O. L.; TEMPORÃO, J. G. A Política Nacional de Promoção da Saúde e a agenda da atividade física no contexto do SUS. Epidemiologia e Serviços a Saúde. 2009; 18(1): 79-86. Disponível em: < http://dx.doi.org/10.5123/S1679-49742009000100008>. Acessado em 31 de maio de 2017.

MELlER, F. O.; CIOChETTO, C. R.; SANTOS, L. P.; DUVAL, P. A.; VIEIRA, M. F. A.; SCH:AFER, A. A. Associação entre circunferência da cintura e índice de massa corporal de mulheres brasileiras: PNDS 2006. Ciência e saúde coletiva. 19(1): 75-82, 2014. Disponível em: < DOI: 10.1590/1413-81232014191.2000. 
MICHAEL, J. W.; SCHLÜTER-BRUST, K. U.; EYSEL, P. The epidemiology, etyology, diagnosis, and treatment of osteoarthritis of the knee. Dteusches Ärzteblatt International. 107(9): 152-62, 2010. Disponível em: <doi: 10.3238/arztebl.2010.0152>.

MUSUMECI, G.; AIELLO, F. C.; SZYCHLINSKA, M. A.; DI ROSA, M.; CASTROGIOVANNI, P.; MOBASHERI, A. Osteoarthritis in the XXIst Century: Risk Factors and Behaviours that Influence Disease Onset and Progression, International Journal Molecular Science. 1(6), 6093-6112, 2015. Disponível em: <doi: $10.3390 / \mathrm{ijms} 16036093$.

OGUNBODE, A. M.; ADEBUSOYE, L. A.; OLOWOOKERE, O. O.; ALONGE, T. O. Physical Functionality and self-rated health status of adult patients with knee osteoarthritis presenting in a primary care clinic. Ethiopian Journal of Health Science. 24(4), 2014. Disponível em: < DOI: http://dx.doi.org/10.4314/ejhs.v24i4.7>.

OLIVEIRA, M. M.; DAHER, D. V.; SILVA, J. L. L.; ANDRADE, S. S. C. A. A saúde do homem em questão: busca por atendimento na atenção básica de saúde. Ciência e saúde coletiva. 20(1): 273-78, 2015. Disponível em: <http://dx.doi.org/10.1590/1413-81232014201.21732013>.

PEREIRA, C. M.; SILVA, A. L. Obesidade e Estilos de Vida Saudáveis: Questões Relevantes para a Intervenção. Psicologia, Saúde \& Doenças. 12(2): 161- 82, 2011. Disponível em: <http://www.scielo.mec.pt/pdf/psd/v12n2/v12n2a01.pdf>.

REZENDE, U. M.; CAMPOS, G. C.; DE PAILO, A. F. Conceitos atuais em osteoartrite. Acta ortopédica brasileira. 21(2): 120-22, 2013. Disponível em: < http://www.scielo.br/pdf/aob/v21n2/a10v21n2.pdf>. Acessado em 31 de maio de 2017.

SILVA, F. A.; SILVA, I. R. Sentidos de saúde e modos de cuidar de si elaborados por homens usuários de Unidade Básica de Saúde - UBS. Revista Ciência e Saúde Coletiva. 19(2): 417-28, 2014. Disponível em: < DOI: 10.1590/141381232014192.04712013

SOWERS, M. F. R; CARRIE, A. K. G, The evolving role of obesity in knee osteoarthritis, Current Opinion Rheumatology. 22(5): 533-37, 2010. Disponível em: < doi: 10.1097/BOR.0b013e32833b4682

TURI, B. C.; CODOGNO, J. S.; FERnANDES, R. A.; MONTEIRO, H. L. Prática de atividade física, adiposidade corporal e hipertensão em usuários do Sistema Único de Saúde. Revista brasileira de epidemiologia. 17(4), 925-37, 2014. Disponível em: < DOI: $10.1590 / 1809-4503201400040011>$.

VELOSO, H. J. F.; SILVA, A. A. M. Prevalência e fatores associados à obesidade abdominal e ao excesso de peso em adultos maranhenses. Revista Brasileira de Epidemiologia.13(3):400-12,2010. Disponível em: http://www.scielo.br/pdf/rbepid/v13n3/04.pdf>. Acessado em 31 de maio de 2017.

WHO - WORLD HEALTH ORGANIZATION. Physical status: the use and interpretation of anthropometry. Report of a WHO Expert Committee. Technical Report Series. 
Geneva;1995.

Disponível

em:

<http://www.who.int/childgrowth/publications/physical_status/en/index.html>. Acessado em: 31 mai. 2017.

WHO - WORLD HEALTH ORGANIZATION. Global status report on noncommunicable diseases Geneva: 2010. World Health Organization, 2011. Disponível em:< http://apps.who.int/iris/bitstream/10665/44579/1/9789240686458_eng.pdf>. Acessado em: 31 mai. 2017. 